\title{
融入统筹理念的分析化学实验教学
}

\author{
姚奇志 $1,2,{ }^{*}$, 金谷 ${ }^{1}$, 李玲玲 1,2 , 李娇 1,2 , 刘红瑜 1,2 \\ 1 中国科学技术大学化学与材料科学学院, 合肥 230026 \\ 2 化学国家级实验教学示范中心(中国科学技术大学), 合肥 230026
}

摘要: 基于一流创新人才培养的要求和分析化学实验课程的特点, 介绍了如何在分析化学实验教学中融入统筹学理 念, 旨在培养学生的统筹能力, 拓展化学实验课程的育人功能, 实现知识技能传授和综合能力培养并重的教学目的, 将创新人才培养的教学理念落到实处。

关键词: 分析化学实验; 化学实验教学; 统筹能力; 创新能力

中图分类号: G64; O6

\section{Integrating the Concept of Planning and Management into the Teaching of Analytical Chemistry Experiment}

\author{
Qizhi Yao ${ }^{1,2, *}$, Gu Jin ${ }^{1}$, Lingling Li ${ }^{1,2}$, Jiao Li ${ }^{1,2}$, Hongyu Liu ${ }^{1,2}$ \\ ${ }^{1}$ School of Chemistry and Materials Science, University of Science and Technology of China, Hefei 230026, China. \\ ${ }^{2}$ National Demonstration Center for Experimental Chemistry Education (University of Science and Technology of China), \\ Hefei 230026, China.
}

\begin{abstract}
Based on the requirements for the cultivation of first-class innovative talents and the characteristics of analytical chemistry experiment course, this paper introduces how to integrate the concept of planning and management into the teaching of analytical chemistry experiment, so as to cultivate students' overall planning ability, expand the educational function of chemical experiment course, and realize both knowledge and skill teaching as well as comprehensive ability cultivation. Finally, the education concept for the cultivation of innovative talents will be implemented.
\end{abstract}

Key Words: Analytical chemistry experiment; Chemistry experiment teaching; Overall planning ability; Innovation ability

分析化学是高校化学类专业的一门重要基础课。欧洲化学协会联合会定义分析化学是一个发展 和应用各种方法、仪器及策略, 以获得物质在特定时间和空间有关组成和性质信息的科学分支。这 就决定了分析化学是一门实验性强和应用范围广的学科, 涉及到化学、物理学、生物学、地质学、 环境科学、医疗和食品等学科, 与人们的日常生活和生产实践息息相关。分析化学实验是分析化学 的重要组成部分, 它是将一系列实验操作与物质的组成、性质融为一体、理论联系实际的综合性课 程。因此, 分析化学实验对学生理论知识运用, 实践技能和综合能力的培养具有重要作用, 是实施

收稿: 2021-07-01; 录用: 2021-07-22; 网络发表: 2021-09-01

*通讯作者, Email: qzyao@ustc.edu.cn

基金资助: 安徽省高等学校省级质量工程重点教研项目(2018jyxm1021); 中国科学技术大学研究生教育创新计划教学改革研究项目(2020ycjg04); 中国科学技术大学本科质量工程项目(2020xjyxm066, 2020xy1kc011) 
素质教育, 培养创新人才, 促进知识向能力转化的重要平台。分析化学实验也具有内容多、操作步 骤复杂、单元教学耗时长的特点, 每一个实验项目涉及玻璃仪器洗涤、试剂称取、溶液配制、溶液 标定和试样溶液的滴定, 甚至试样的各种前处理等一系列过程。分析化学实验课程的这些特点为开 展统筹学理念教学提供了多维度空间。近年来, 我们在日常实验教学过程中, 通过融入统筹学方法, 指导学生科学合理地设计实验过程, 有条不紊地开展实验, 不仅提高了实验课的效率和学生实验预 习的自觉性, 实现知识技能传授和统筹能力培养, 而且也促进了学生对实验内容的理解和掌握, 提 升了学生的实验能力和综合素质, 取得了事半功倍的教学效果。

\section{1 统筹学中的统筹方法介绍}

统筹学是一门现代科学, 是应用数学来研究和分析一项工作的合理组织与安排的科学。统筹学 作为一门用来解决实际问题的学科, 已经深入到日常生活中。数学家华罗庚以日常生活中的烧水泡 茶为例给出了很好的说明: 想泡茶喝, 没有开水, 水壶没洗, 茶壶、茶杯也没洗; 火生了, 茶叶也 有了。怎么办？泡茶各项准备工作所需时间见图1。

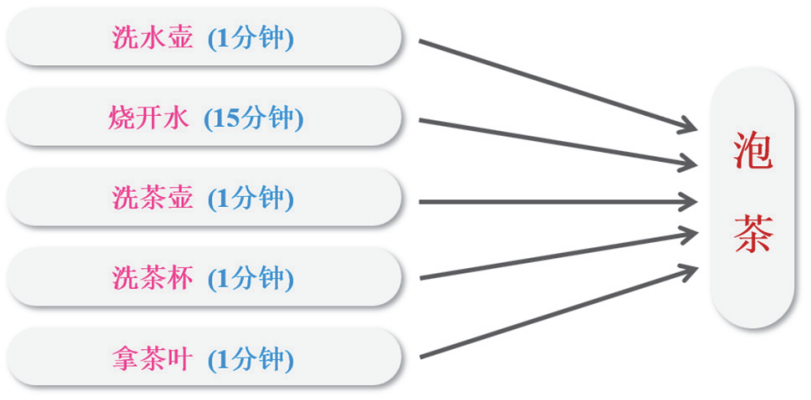

图1 泡茶前所需准备工作时间

方法甲：洗水壶，灌水烧水；洗茶壶、洗茶杯、拿茶叶; 水开，泡茶喝。

方法乙：洗水壸, 洗茶壸、洗茶杯, 拿茶叶, 灌水烧水; 水开, 泡茶喝。

方法丙: 洗水壸, 灌水烧水; 水开; 拿茶叶, 洗茶壶、洗茶杯, 泡茶喝。

哪一种方法省时高效? 很明显第一种方法更加科学合理, 甲在 $16 \min$ 就可以喝茶, 而乙、丙则 需要 $19 \min ^{[1]}$ 。甲很好地规划准备工作节奏, 利用烧开水所需 $15 \mathrm{~min}$ 的等待时间清洗茶壶、茶杯、拿 茶叶, 所以水开即可泡茶。华罗庚先生用日常生活中小事例生动地给我们阐释了统筹学的应用。

\section{2 分析化学实验课程特点}

\section{1 数据要求严格, 器皿洁净度高}

分析化学实验是为了获得被测物的浓度、含量等信息, 侧重培养学生 “量” 的概念。化学分析 要求学生实验结果的误差不超过 $6 \% 0-8 \%$ 。这就要求实验过程中所用到的器血必须用自来水清洗干 净, 并用蒸馏水润洗 3 遍, 部分容量器血须用操作液润洗 3 遍, 才能满足误差操作要求。因此, 分析 化学实验中各类器血的洗涤用时长。

\section{2 操作步骤繁多, 实验耗时长}

为了确保实验结果的精密度和准确度, 不论标准溶液标定, 还是样品的滴定, 每位同学必须至 少平行做 3 份, 实验任务重, 操作步骤复杂, 实验所需时间长。

\section{3 前处理过程复杂, 操作要求高}

从配位滴定实验开始均有复杂的试样前处理过程, 前处理过程涉及到试样加热, 调控溶液 $\mathrm{pH}$ 等 操作, 需要用到通风柜、电热板等公用设备。而且该过程不仅要准确控制样品溶液 $\mathrm{pH}$, 对操作速度 也有严格要求。例如, 无录盐法测定铁矿石中全铁含量的预还原步骤, 不仅要求控制好温度, 确保 
溶液中 $\mathrm{Fe}^{3+}$ 完全被还原, 而且要求尽可能快完成预还原操作, 减小空气中的 $\mathrm{O}_{2}$ 对还原产物的影响。

\section{4 操作要求规范, 确保数据准确度}

鉴于分析实验对数据结果的严格要求, 学生实验过程中的关键操作步骤必须严格按照规范操作 进行。规范操作是数据准确的根本保证, 任何不规范的小失误都可能带来比较大的误差, 导致实验 失败。

\section{3 我校分析化学实验教学现状}

经过二十余年教学改革和实践, 我校分析化学实验教学水平和效果稳步提升。如今在实验课堂 上, 学生 “照方抓药” 的现象明显改善。实验前能够针对实验内容和步骤, 认真预习和思考; 实验 过程中, 带着自己的思考进行实验, 遇到异常现象能先思考再讨论、请教。在综合、探究、设计型 实验项目中, 能运用所学理论知识展开深入探究, 并发表课程研究相关的教学论文 ${ }^{[2-4]}$ 。尽管如此, 但在教学实践中, 我们也发现学生对整体实验内容的把控能力和实验过程统筹尚存不足, 实验过程 中匀匀忙忙、丢三落四, 实验仪器摆放凌乱、台面不整洁等现象时常发生, 甚至有的学生在规定的 实验课时内, 无法完成实验。这些说明虽然学生掌握了基本操作规范, 分析和解决问题能力也得到 提升, 但对实验步骤安排、实验时间分配、实验仪器布局等不够合理, 统筹能力欠缺。

\section{4 分析化学实验与统筹方法}

\section{1 教学内容的统筹安排}

根据知识体系循序渐进的原则, 我校秋季学期分析化学实验安排如表 1 所示 ${ }^{[5]}$ 。首先是基本操作 练习和简单的酸碱滴定, 逐步过渡到需要预处理控制溶液 $\mathrm{pH}$ 的配位滴定, 发展到既有复杂预处理又 有滴定条件控制的氧化还原滴定，最后完成自主探究实验和开放设计实验。

表1 分析化学实验项目安排

\begin{tabular}{|c|c|c|}
\hline 实验项目 & 类型 & 学时数 \\
\hline 安全教育, 基本操作练习 & 操作练习 & 8 \\
\hline 未知碱浓度测定 & 酸碱滴定 & 8 \\
\hline 有机酸摩尔质量测定 & 酸碱滴定 & 8 \\
\hline 返滴定法测定明矾中铝含量 & 配位滴定 & 8 \\
\hline 碘量法测铜合金中铜的含量 & 氧化还原滴定 & 8 \\
\hline 无录盐法测定铁矿石中全铁含量 & 氧化还原滴定 & 8 \\
\hline 白云石中钙含量测定 & 沉淀制备及氧化还原滴定 & 8 \\
\hline $\mathrm{BaSO}_{4}$ 反应历程探究 & 自主探究型 & 8 \\
\hline 自主设计实验 1 & 自主开放设计型 & 8 \\
\hline 自主设计实验2 & 自主开放设计型 & 8 \\
\hline
\end{tabular}

基于该层次化、递进式教学体系, 我们在 “安全教育, 基本操作练习” 单元, 清楚交代实验室 的空间布局、试剂摆放规律, 通风柜、电热板等公用设备使用要求, 使学生对实验环境及硬件设施 有总体的了解, 以便后续进行统筹安排。“未知碱浓度测定” 单元, 通过介绍著名的 “华罗庚烧水泡 茶” 统筹法, 引入统筹理念; 在 “有机酸摩尔质量测定” 实验中, 通过对具体实验环节统筹实例讲 授, 向学生展示统筹规划的优越性。后续实验中, 要求学生在预习时, 按照实验内容和步骤, 运用 统筹学方法, 设计出具体实验流程。借助现代多媒体技术, 通过翻转课堂的教学模式, 线上指导和 线下讨论相结合, 逐步将统筹学方法引入实验课堂教学中, 培养学生的统筹能力, 拓展实验课教学 的育人功能。 


\section{2 统筹方法引入实验教学实例}

以 “有机酸摩尔质量测定” 实验中 $\mathrm{NaOH}$ 溶液标定步骤为例。原教材中实验步骤: 配制邻苯二甲 酸氢钾标准溶液于 $250 \mathrm{~mL}$ 容量瓶中, 准确移取 $25.00 \mathrm{~mL}$ 于锥形瓶进行标定, 平行标定 3 份。为了便于 统筹方法教学, 我们将移液标定设计为单份称样标定, 具体步骤如下: 准确称取 0.4-0.6 g邻苯二甲 酸氢钾于 $250 \mathrm{~mL}$ 锥形瓶中, 加 $25 \mathrm{~mL}$ 蒸馏水, 溶解完全后, 滴加 2 滴 $0.5 \%$ 的酚唒指示剂, 用标准 $\mathrm{NaOH}$ 溶液滴定至出现稳定粉红色半分钟不褪去, 根据消耗 $\mathrm{NaOH}$ 溶液体积计算出 $\mathrm{NaOH}$ 溶液准确浓度, 平 行标定 3 份, 要求精密度小于 $2 \%$ 。此过程中, 由于邻苯二甲酸氢钾晶体颗粒较大, 完全溶解需要时 间或者加热, 对该过程的处理可以有多种方法。图2给出了标定前各项准备工作所需的预估时间。课 堂上, 通过提问、讨论, 同学们列举了多种可能的途径。

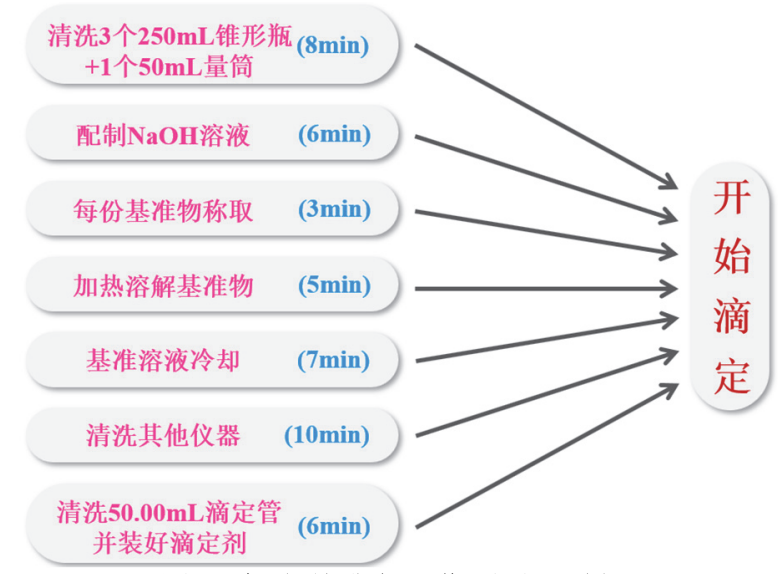

图2 标定前准备工作所需要时间

方法甲: 首先清洗所有实验所需仪器, 然后配制 $\mathrm{NaOH}$ 溶液, 并将溶液加入滴定管; 用托盘托着 3 个雉形瓶, 带好记录本到天平室, 分别称取邻苯二甲酸氢钾于各雉形瓶中, 用量筒各加入 $25 \mathrm{~mL}$ 蒸 馏水; 放在电热板上加热至完全溶解, 放置在实验台面冷却至室温, 开始滴定; 耗时61 min。

方法乙: 先洗三个雉形瓶和量筒, 分别称取邻苯二甲酸氢钾于各雉形瓶内, 用量筒各加入 $25 \mathrm{~mL}$ 蒸馏水, 摇匀放置在实验台; 清洗其他仪器, 配制 $\mathrm{NaOH}$ 溶液, 并将溶液加入滴定管; 此时, 雉形瓶 里基准物已经完全溶解, 开始滴定; 耗时 $23 \mathrm{~min}$ 。

方法丙: 清洗所有实验所需仪器; 配制 $\mathrm{NaOH}$ 溶液, 并将溶液加入滴定管; 拿着 1 个雉形瓶和记 录本到天平室, 称取邻苯二甲酸氢钾于雉形瓶内, 用量筒加入 $25 \mathrm{~mL}$ 蒸馏水, 放在电热板上加热至完 全溶解, 放置在实验台面冷却至室温, 开始滴定; 再称取第2份基准物..... ; 耗时超过 85 min。

显然, 方法乙的操作, 科学地运用了统筹方法, 对实验内容进行了合理的安排和分配, 充分利 用溶解基准物所需要的时间进行其他准备工作, 仅 $23 \mathrm{~min}$ 就可以开始进行滴定。同样的工作量, 方 法甲的操作中, 没有合理地利用溶解样品所需时间, 增加了加热溶解及溶液冷步骤, $61 \mathrm{~min}$ 才能开 始滴定。方法丙则照搬实验教科书, 没有自己的思考, 滴定前的准备工作耗时超 $85 \mathrm{~min}$ 。

通过该课程教学案例讲授和讨论, 使学生了解统筹方法是科学利用时间和资源的艺术。融入统 筹方法的实验过程, 如同指挥一场战役, 需要运筹帷幄, 提前布局, 按计划各 “兵种” 协同配合。

\section{3 融入统筹方法的实验教学成效}

(1) 内化了实验内容: “学而不思则罔, 思而不学则殆”。融入统筹学理念的实验教学, 学生在 实验预习阶段, 首先要了解实验目的和原理, 再结合所学理论知识, 厘清各实验步骤之间的相互关 系和相互影响因素。哪些步骤可以同时进行, 哪些步骤需要提前完成, 哪些试剂只能现配现用等。 只有这样, 才有可能对实验步骤进行有效的整合, 设计出合理的实验流程图。此过程不仅训练学生 的统筹能力, 更加深了学生对实验内容的掌握和理解, 实现所学知识的内化。 
(2) 培养了学以致用的实践能力: “纸上得来终觉浅, 绝知此事要躬行”。融入统筹方法教学后, 要求学生在实验前, 规划设计实验流程, 该环节既检验了学生对理论知识的理解和掌握, 也为学生 运用理论知识解决实际问题提供了机会, 培养他们学以致用的实践能力。例如: “返滴定法测定明 矾中铝含量” “无录盐法测定铁矿石中全铁含量” 两个实验 (表1)滴定前的预处理环节均需要加热操 作。在铝含量测定实验中, 样品溶液的加热预处理可以同时进行, 但同样的操作在全铁测定实验中 则不能生搬硬套, 因为预还原产物 $\mathrm{Fe}^{2+}$ 易被空气中的 $\mathrm{O}_{2}$ 氧化, 3份样品同时处理, 则会产生比较大的 误差, 导致实验失败, 所以学生设计流程图时, 必须运用所学的氧化还原反应知识进行综合分析。 这不仅是一个主动思考的过程, 更是锻炼学生如何用所学理论知识解决实际问题的能力。

(3) 提高了实验课的教学效率, 提升了学生综合素质: “凡事预则立, 不预则废”。融入统筹方 法的实验教学实践证明, 学生逐渐养成了 “先动脑后动手” 的实验习惯, 培养了主动思考的能力, 实验效率明显提高。在规定的实验课时内, 学生能很好地完成实验任务, 课堂秩序井然有序。不仅 如此, 学生撰写的实验报告条理性、系统性更强, 对实验现象和数据结果的分析讨论更有深度, 综 合实验能力得到提高。因此, 融入了统筹方法的分析化学实验教学培养了学生合理规划的统筹能力, 提高了实验效率, 拓展了教与学的空间, 学生综合素质得到显著提升。

\section{5 结语}

教学实践证明, 将统筹方法融入分析化学教学, 学生实验效率明显提高, 扎堆称取试剂或扎堆 排队预处理, “想到什么做什么” 的混乱现象不见了。取而代之的是学生实验时, 台面整洁, 仪器 摆放有序, 各自有条不紊地完成实验。同时, 学生对实验内容的理解和掌握更加深入, 知识运用能 力明显增强, 实验成就感和实验兴趣显著提升。融入了统筹理念的分析化学实验教学, 不仅培养了 学生良好的思维和实验习惯, 也促使教师不断学习, 完善自身知识、能力体系, 保障分析化学实验 教学质量稳步提升。

\section{参 考 文 献}

[1] 华罗庚. 统筹方法平话及补充. 北京: 中国工业出版社出版, 1966.

[2] 范天娇, 姚奇志, 金谷. 大学化学, 2021, 36 (2), 1912054.

[3] 姚奇志, 李玲玲, 金谷, 刘红瑜, 李娇. 大学化学, 2022, 37 (1), 2103040.

[4] 向思佳, 廖光旭, 姚奇志, 金谷, 李玲玲. 大学化学, 2021, 36 (9), 2012053.

[5] 金谷, 姚奇志, 江万权, 李娇, 刘红瑜, 王晓葵. 分析化学实验. 第2版. 合肥: 中国科学技术大学出版社, 2020 . 